\title{
Porcine reproductive and respiratory syndrome virus dissemination across pig production systems
}

2 in the United States

3

4 Running title: Evolutionary epidemiology of PRRSV

5 Manuel Jara ${ }^{1}$, David A. Rasmussen ${ }^{2,3}$, Cesar A. Corzo ${ }^{4}$, Gustavo Machado ${ }^{1 *}$

$6 \quad{ }^{1}$ Department of Population Health and Pathobiology, College of Veterinary Medicine, North Carolina

7 State University, Raleigh, NC, USA.

${ }^{2}$ Department of Entomology and Plant Pathology, North Carolina State University, Raleigh NC, USA

${ }^{3}$ Bioinformatics Research Center, North Carolina State University, Raleigh NC, USA

${ }^{4}$ Veterinary Population Medicine Department, College of Veterinary Medicine, University of Minnesota, 1365 Gortner Ave, St Paul, MN 55108, USA.

*Corresponding author: gmachad@ncsu.edu

\section{Summary}

Porcine reproductive and respiratory syndrome virus (PRRSV) remains widespread in the North American pig population. Despite improvements in virus characterization, it is unclear whether PRRSV infections are a product of viral circulation within a farm, within production systems (local) or across production systems (external). Here we examined the dissemination dynamics of PRRSV and the processes facilitating its spread within and among pig farms in three production systems. Overall, PRRSV genetic diversity declined since 2018, while phylodynamic results support frequent transmission acrossproduction systems. We found that PRRSV dissemination occurred mostly through transmission between farms of different production companies, which were predominant for several months, especially from November until May when PRRSV tends to peak in the studied region. Within production systems, dissemination occurred mainly through regular pig flow (from sow to nursery and then to finisher farms); nevertheless, an important flux of PRRSV dissemination from finisher to sow and nursery farms highlighted the importance of downstream farms as sources of the virus. Farms at areas with pig density from 500 to $1000 \mathrm{pig} / \mathrm{km}^{2}$ and farms located at a range within $0.5 \mathrm{~km}$ and $0.7 \mathrm{~km}$ from major roads were 
more likely to infect by PRRSV, whereas farms at elevation between 41 and 61 meters and denser vegetation acted as dissemination barriers. Although remains a challenge, there is a need to disentangle the route of PRRSV transmission, results evidenced that dissemination among commercially unrelated pig production systems was intense, reinforcing the importance of farm proximity on PRRSV spread. Thus, consideration of farm location and their geographic characteristics may help to forecast dissemination.

32 The understanding of PRRSV transmission routes has the potential to inform targeted strategies for its

33 prevention and control. Further studies are needed to quantify the relative contribution of PRRSV

34 transmission routes.

35 Keywords: molecular epidemiology, phylodynamics, virus dispersal, between-farm dissemination.

Introduction

38 Porcine reproductive and respiratory syndrome virus (PRRSV) remains among the most costly diseases in

39 North America (Neumann et al., 2005; Holtkamp et al., 2013; Pileri and Mateu, 2016). Despite the great

40 advances in reducing the incidence of PRRSv in some regions (Rathkjen and Dall, 2017) by enhanced

41 biosecurity (Silva et al., 2019) and immunization strategies (Corzo et al., 2010), every year approximately

$42 \quad 20-30 \%$ of US breeding herds still become infected with PRRSV (MSHMP, 2020).

PRRS is caused by an enveloped positive single-stranded RNA virus in the family Arteriviridae,

44 order Nidovirales (Cavanagh et al., 1990). The genome of PRRSV is $\sim 15 \mathrm{~kb}$ and consists of 9 open

45 reading frames (ORFs) that encode seven structural proteins and 14 non-structural (Dokland, 2010). The

46 mutation rate of PRRSV hinders the effectiveness of immune response against newly introduced variant

47 strains, especially in farms with recurrent outbreaks (Linhares et al., 2014; Sanhueza et al., 2019).

48 Likewise, recombination between North American wild-type virus strains and modified-live vaccines

49 represents an important mechanism in the historical evolution and spread of PRRSV (Yuan et al., 1999).

50 The phylodynamic patterns of PRRSV spread and dissemination among pig systems and farm

51 types have been previously described (Shi et al., 2010; Franzo et al., 2015; Alkhamis et al., 2016;

52 Alkhamis et al., 2017; Sun et al., 2019). While there was support for between-production system 
dissemination (Alkhamis et al., 2016), the relevance of transmission in the maintenance of local

54 incidence, in comparison with within-production system circulation, remains unknown. Thus, the

question of whether PRRSV circulation is the product of local dissemination between farms that are part

of a common commercial network (production system) versus the result of external viral introductions

57 from nearby infected farms of distinct production systems still remains (Arruda et al., 2018).

Bayesian phylodynamic approaches have provided a breakthrough in tracking pathogen

dissemination dynamics (Baele et al., 2018; Faria et al., 2019; Hicks et al., 2020). Structured coalescent

61 dissemination versus external introductions to the overall incidence of disease (Rasmussen et al., 2018;

62 Yang et al., 2019; Hicks et al., 2020). Novel methods have been developed to integrate spatial and or external introductions and identified the factors that facilitate or impede PRRSV spread.

\section{$70 \quad$ Material and methods}

\section{$71 \quad$ Data collection}

72 In total, our dataset comprises 4,970 ORF-5 sequences collected from 2014 to 2019, obtained from three

73 commercially unrelated pig production systems (here coded as A, B, and C), in the US. Production

74 system A, B and C do not shared feed truck or traded pig among themselves. Sequences were generated

75 through outbreak investigation and from regular surveillance activities performed by each production

76 system and shared with the Morrison Swine Health Monitoring Program (MSHMP). Each sequence was

77 attributed to a farm type (e.g., sow, nursery, finisher, and boar stud), geographic location, pig production

78 system (A, B, and C), and the date when the sequence was reported. 


\section{Preliminary phylogenetic analysis}

80 Sequences were aligned using Mega X, available at www.megasoftware.net (Kumar et al., 2018). The

81 recombination detection program (RDP) v5.3 was used to search for recombination within our dataset

82 (Martin et al., 2015). The alignment was screened using five methods (BootScan, Chimaera, MaxChi,

83 RDP, and SiScan), evidencing 63 recombinant sequences, which were removed from the downstream

84 analysis (Table S1). To determine whether there was a sufficient temporal molecular evolutionary signal,

85 we used TempEst v1.5 (Rambaut et al., 2016). To calculate the $P$-values associated with the phylogenetic

86 signal analysis, we followed Murray et al. (2016) using 1,000 random permutations of the sequence

87 sampling dates (Navascués et al., 2010). The relationship found between phylogenetic tree root-to-tip divergence and sampling dates (years) supported the molecular clock analysis for this study $(P<0.05)$.

\section{Discrete and continuous phylogeographical analysis}

Phylogenies were estimated by Bayesian inference through Markov chain Monte Carlo (MCMC), implemented in BEAST v2.5.0 (Bouckaert et al., 2014). By using ModelFinder (Kalyaanamoorthy et al., across systems and between farm types (i.e., sow, nursery, finisher, boar stud), we used the discrete-trait

97 phylogeographic models implemented in BEASTv2.5.0 to reconstruct ancestral states (Bouckaert et al., 2014). To explore the most important historical dissemination routes for PRRSV spread across farms, as

99 well as among pig systems, we used Bayesian stochastic search variable selection (BSSVS) (Lemey et al.,

100 2009). Through BSSVS, we identified the non-zero rates of change between each pair of discrete traits

101 (production system and farm types) based on its Bayes factor value (lower than 3). To perform this

102 analysis, an asymmetric migration rate matrix was assumed. To infer the intensity of directional

103 transitions (forward and backward) within the migration matrix, we used a Markov jump approach. To

104 evaluate oversampling and possible selection bias towards location and/or farm types with 
disproportionate amounts of samples (i.e., sow farms), we downsampled on each system to match the number of samples between the three systems, maintaining the same proportion of farm type (system A $89 \%, \mathrm{~B}=-39.5 \%$, and $\mathrm{C}=31.4 \%$ ).

To analyze the spatiotemporal spread of PRRSV, we performed a Bayesian continuous phylogeographic analysis, where the nucleotide substitution model was similar to the discrete model

110 mentioned above. In addition, we used an uncorrelated relaxed molecular clock model with lognormal

111 distribution (Drummond et al., 2006). The coalescent tree prior used was Bayesian SkyGrid with 112 covariates (Gill et al., 2016). Phylogeographic inference of ancestral locations was performed under a 113 relaxed random clock model, considering a lognormal distribution. All analyses were run for 200 million 114 generations, sampling every 10,000 th generation and removing $10 \%$ of the chain as burn-in. To estimate

115 the relative genetic diversity, we used skygrid which is based on a nonparametric coalescent model, and is utilized to calculate the effective population size over time (Hill and Baele, 2019). To visualize the

117 spatiotemporal spread of PRRSV, we used Spatial Phylogenetic Reconstruction of Evolutionary

118 Dynamics using Data-Driven Documents (D3) SPREAD3 software (Bielejec et al., 2016). To evaluate the

119 impact generated by the imbalance distribution of sequences among systems and production types, a

120 sensitivity analysis was performed. A subsample taken from the systems and farm types with the highest

121 number of sequences was used to match the sample size in the systems with the least number of

122 sequenced, thus this smaller sample was reanalyzed (Supplementary material S6).

\section{Spatiotemporal epidemiological statistics}

124 To summarize PRRSV diffusion over time and space we analyzed data from each production system

125 separately and all the sequences as a whole, using the R package "seraphim" version 1.0 (Dellicour et al.,

126 2016). Spatiotemporal information about lineage locations was extracted from 100 input trees sampled at

127 regular intervals from the post-burn-in posterior distribution to account for phylogenetic uncertainty

128 (Dellicour, Rose and Pybus, 2016). Each phylogeny branch was summarized and represented as a distinct 129 vector defined by its starting geographic location, ending geographic location and dates (Dellicour, Rose and Pybus, 2016; Dellicour et al., 2016). Each branch vector represents an independent viral lineage 
131 dissemination event (Pybus et al., 2012; Laenen et al., 2016). Each vector was assigned to a diffusion

132 coefficient value $(D)$ for subsequent visualization: as described by (Pybus et al., 2012), which accounts

133 for the time (in years) during which each specific vector (lineage) moved from its initial location until the

134 end location of each lineage (measured from the location of the tree root). These vectors were also used to

135 assess the invasion velocity of the sampled lineages. To determine and compare the PRRSV diffusivity

136 associated with the overall data and for each production system, we estimated the spatial diffusion

137 coefficient using $D$-weighted statistics (Trovão et al., 2015) since this index is less sensitive to extreme

138 values of short branches. These results were displayed via: i) dissemination epidemiological statistics that

139 estimated the mean branch dissemination velocity, and ii) kernel plots showing the lineage diffusion

140 coefficients of the mean and variation on the highest posterior density (HPD).

Between-production systems transmission: the contribution of within-system PRRSV circulation

143 We estimated the contribution of local transmission (i.e., between farms within a production system)

144 versus external introductions (i.e., between farms across production systems) using a structured

145 coalescent model. Due to computational demand, we used PRRSV alignment from MEGA software

146 (Kumar et al., 2008) to produce a phylogenetic tree using PhyML version 3.0 (Guindon et al., 2010),

147 using a substitution rate $=0.00672$ (estimated), and considering an HKY Hasegawa-Kishino-Yano $+\Gamma 4$

148 (Hasegawa et al., 1985; Yang, 1994), which was dated using Least Squares Dating (LSD) version 0.3 (To

149 et al., 2016). To infer the contribution of local versus external introductions the migration rates between

150 different pig production systems were estimated using the Marginal Approximation to the Structured

151 Coalescent (MASCOT) package (Müller et al., 2017, 2018) implemented in Beast2 (Bouckaert et al.,

152 2014). To infer the evolutionary rate from the time-calibrated tree, we used a strict clock model. Using

153 MASCOT, we estimated the effective population size in the local and external population and the

154 migration rates between them, which was allowed to be asymmetric. This analysis was replicated three

155 times, each time using one of the three pig systems as local and the other two as external. We also 
calculated the posterior probability of each internal node in the tree being in each state (i.e., local or external) using the forwards/backwards approach implemented in MASCOT (Müller et al., 2018).

All analyses were performed under 100 million MCMC runs, states were sampled every 10,000 and the

159 first $10 \%$ of samples were discarded as burn-in using TreeAnnotator v. 2.3.0 (Rambaut and Drummond,

160 2016). We reconstructed the number of migration events through time using the ancestral locations

161 reconstructed by MASCOT. A migration event was assumed to occur when the most probable location of

162 a lineage changed between its parent and child node, with the time of the migration event assumed to be

163 the date of the daughter node. Migration events (local and external) per month were plotted using "baltic"

164 (https://github.com/blab/baltic) in Python Software Foundation version 3.7, available at

165 http://www.python.org. Finally, we compared the differences in local vs external transmission between

166 PRRSV season and off-season through two-sample Wilcoxon test (Hogg et al., 2010), PRRSV season was

167 determined by the exponentially weighted moving average proposed by MSHMP (MSHMP, 2020). In the

168 same way as we described in the section above, a sensitivity analysis with a subsample was evaluated for

169 consistency of the results (Supplementary material S8).

\section{Factors promoting or restricting PRRSv spread}

171 To determine the effect of different predictors on the spread of PRRSV, we analyzed the results of the

172 continuous phylogeographic analysis using the R package "seraphim" version 1.0 (Dellicour et al., 2016),

173 considering a continuous location approach (based on the geographic coordinate location of each pig

174 farm) and a random walk diffusion model. Spatiotemporal information was extracted from 100 trees

175 sampled at regular intervals from the posterior distribution, after burn-in, to account for phylogenetic

176 uncertainty. Each phylogenetic branch was considered a vector defined by its start and end location (e.g.

177 latitude and longitude), and its start and end dates. Statistical significance for the correlation between

178 phylogenetic and predictive factors was tested using 100 trees generated and expressed in the form of

179 Bayes factors (BF) (Dellicour et al., 2016). Based on the extracted spatio-temporal information contained

180 in each phylogenetic tree described above, we calculated the distances based on predictor variables

181 associated with every branch of each tree, which were used to determine the correlation between the 
duration of each branch in the phylogeny and its related predictor variable distance, including the annual temperature, annual precipitation, elevation, runoff (an index that quantity of water discharged in surface streams), soil humidity (commonly used to quantify topographic control on hydrological processes) (Sørensen et al., 2005), enhanced vegetation index (EVI), which can be used to quantify vegetation greenness, as well as the pig density, and distance to the main roads (Fig. 1 and Supplementary Table S3

187 for more details). Briefly, the distance between each farm (geographic coordinates) to the nearest main 188 road was calculated based on the Euclidean distance. We calculated the statistic $Q$, which represents how much variation in lineage movement is explained with spatial heterogeneity when each predictor variable is considered (Dellicour, Rose and Pybus, 2016). Here, $Q=\mathrm{R}^{2}{ }_{\text {env }}-\mathrm{R}^{2}{ }_{\text {null }}$ where $\mathrm{R}^{2}$ env is the coefficient of determination obtained from the regression between branch duration against predictor (factors) distances, while $\mathrm{R}_{\text {null }}^{2}$ represents the coefficient of determination for the regression between branch duration against predictor distances, which were replaced by a null raster. Thus, the factors that were considered for

194 further Bayes factor (BF) analysis, were the ones that obtained positive $Q$ values in at least $90 \%$ of the tested trees (Jacquot et al., 2017). We approximated a BF value for all predictors tested that passed the above-mentioned $Q$ statistic. Predictive factors were treated as a conductance (variables that promote the

197 spread of the disease) or a resistance factor (variables that impede its spread). For the interpretation of BF, 198 values between 3-20 were considered "positively" supported, values between 20-150 were considered 199 "strong", and values >150 were considered " overwhelming support" (Kass and Raftery, 1995). For all variables that showed a significant support either as conductance of resistance factors (considering $Q$ 201 statistic and Bayes factor) (Supplementary Table S10), each raster was subdivided into four alternative

202 ranges based on Jenks natural breaks, for example, for elevation raster values below 20 meters constituted 203 the low elevation areas, from 21-40 meters medium-low elevation, 41-60 medium-high elevation and $>61$ 204 high elevation (Supplementary Table S11). The same continuous phylogeographic analysis using the R 205 package "seraphim" was applied individually for each range raster as detailed above.

\section{Results}

\section{Study population}


A total of 63 recombinant PRRSV sequences were identified and removed from the database. Most recombinants $(52 \%)$ belonged to system $\mathrm{B}$ with the highest number of recombinants $(82 \%)$ belonging to sow farms (Supplementary Table S1). Thus, 4,753 sequences remained for further discrete and continuous

211 phylogeographic analyses. Most sequences originated from system A = 3,748 (79\%), while B had 626

212 (13\%), and $\mathrm{C}$ had $379(8 \%)$. Overall, 2,958 sequences $(62.2 \%)$ belonged to sow farms, $909(19.1 \%)$ to

213 finishers, $873(18.4 \%)$ to nurseries, and $13(0.3 \%)$ to boar studs (Supplementary Table S1). The nearest-

214 neighbor distances among farms of distinct production systems had a median value of $7.6 \mathrm{~km}(1 \mathrm{st}$

215 quartile $=3.6 \mathrm{~km}$ and $3 \mathrm{rd}$ quartile $=19.3 \mathrm{~km}$ ), the closest related production systems were $\mathrm{A}$ and $\mathrm{C}$ with

216 median distance among all pairwise farms of $1.8 \mathrm{~km}(1 \mathrm{st}$ quartile $=1.2 \mathrm{~km}$ and $3 \mathrm{rd}$ quartile $=2.5 \mathrm{~km})$, and

217 finisher farms of system $\mathrm{A}$ and $\mathrm{C}$ were the closest with median of $1.9 \mathrm{~km}(1 \mathrm{st}$ quartile $=1.3 \mathrm{~km}, 3 \mathrm{rd}$

218 quartile $=3.2 \mathrm{~km})($ Supplementary Table S4 and S5).

219 Discrete phylogeography analysis

220 The results of PRRSV ancestral reconstruction showed that the most likely center of origin was

221 production system A with a root state posterior probability $(\mathrm{RSPP})=0.84$, coming from a sow farm

$222(\mathrm{RSPP}=0.75)$. From there, it spread heterogeneously across the other systems, unrestricted to farm types

223 (Fig. 2A). The reconstructed phylogenetic relationship did not show evidence of any cluster related to

224 production systems. However, we observed a clear dominance of PRRSV originating from system A over

225 the other systems (Fig. 2A). Our sensitivity analysis on the downsample supported these results

226 (Supplementary Table S6). The relative genetic diversity expressed as the effective population size (e.g.

227 number of individuals contributing to a new PRRSV infection) over time showed a plateau until 2012.

228 After that time, we captured a steep increment that lasted until approximately 2016, when relative genetic

229 diversity decreased through June 2019 (Fig. 2B), the last month of data available for our study. The

230 temporal pattern shown by the sampled PRRSV sequences evidenced the typical annual trend of seasonal

231 outbreaks (Fig. 2B) (MSHMP, 2020). Phylogeographic results of system A showed that its most likely

232 center of origin was a sow farm $(\mathrm{RSPP}=0.47)$, followed by an almost equal probability for origins in

233 finisher farm $(\mathrm{RSPP}=0.27)$ and nursery farm $(\mathrm{RSPP}=0.26)$, the boar stud farms were negligible (RSPP 
$234<0.01)($ Fig. 3A). In recent years, there was an evident increase in the proportion of PRRSV positive

235 finisher farms, reaching a similar level of representation than sow farms for some clusters (Fig. 3A).

236 SkyGrid plot revealed a relatively constant trend of relative genetic diversity until $\sim 2011$, showing a

237 linear increase in population size, which reached its highest levels 2016 (Fig. 3B). We observed a

238 similar trend when we analyzed all systems together (Fig. 3B), 2015 and 2018 were the years with more 239 samples sent to be sequenced (Fig. 3B).

For production system $\mathrm{B}$, a sow farm $(\mathrm{RSPP}=0.4)$ was also the most likely origin; however, a

242 by the effective population size was static until 2012, after which it increased rapidly until 2015-2016,

243 when it started a linear decrease until June 2019, similar to the seasonal pattern shown by the number of

244 sequences (Fig. 4B). As observed in systems A and B, the most likely center of origin for system C

245 occurred inside a sow farm $(\mathrm{RSPP}=0.52)$, followed by a high probability for originating in a finisher

246 farm $(\mathrm{RSPP}=0.4)$. In contrast to the other systems, we observed a low probability of origin related to a

247 nursery farm (RSPP $>0.1$ ) (Fig. 5A). The temporal trend in the number of sampled sequences showed the

248 mentioned annual seasonal pattern, with peak occurrence 2015 . SkyGrid plot evidenced a more stable

249 pattern, with higher levels of variation in effective population size during the period 2013-2014, then

250 decreasing until 2018, when it showed a small peak and then decreased through June 2019 (Fig. 5B).

\section{Phylogeographic diffusion analysis and spread statistics}

252 The reconstructed spatiotemporal diffusion of PRRSV was characterized by continuous expansion in the

253 geographic space, accompanied by diversification events. The comparison between each system and all

254 samples together showed two noticeable patterns. The first one was characterized by a radial expansion

255 over time, found when all samples were analyzed together and within samples of system A. In contrast,

256 systems B and C evidenced a directional trend, since for both systems the spread started in the southwest

257 region. In the case of system B, PRRSV spread to the northeast, while for system C, the spread was

258 mainly to the north, though spread did occur in all directions (Supplementary Figs. S1-3). 
The spatiotemporal analysis showed that when all sequences were analyzed together, the

260

261

262

estimated median value for the mean branch velocity was $39 \mathrm{~km} /$ year $(95 \% \mathrm{HPD}=31.2-61.1)$. This median value was lower than that shown by system A (49.2 km/year, 95\% HPD= 42.9-60.4), and higher than the values observed in systems B and C (32.7 km/year, 95\% HPD= 29.1-43.3), and (26.4 km/year, 95\% HPD $=20.1-41.0)$, respectively. Estimated diffusion coefficients were asymmetric for each system and when all samples were analyzed together. System A showed the highest diffusivity $\left(243.8 \mathrm{~km}^{2} /\right.$ year$)$; however, its diffusion variation among branches was the lowest registered. The overall results (all sequences) evidenced the highest among-branch variation, while its diffusion coefficient $\left(150 \mathrm{~km}^{2} /\right.$ year $)$ was lower than system A and substantially higher than the other systems. Systems B and C showed a similar pattern in terms of diffusion coefficient variation among branches; however, B was higher than C (97 km²/year) and (51 km²/year), respectively (Supplementary Fig. S4).

\section{PRRSv dissemination among pig systems and farm types}

Bayesian stochastic search variable selection (BSSVS) identified that the most significant disseminations were highly related to system A, evidencing strong support by a Bayes Factor score $>20$. The statistical support or evidence for system A sourcing most PRRSV strains to system B, BF $=102.1$ (average relative migration rate "ARMR"= 1.79), followed by dissemination from $\mathrm{A}$ to $\mathrm{C}, \mathrm{BF}=47.8(\mathrm{ARMR}=0.46)$; of less importance was from $\mathrm{B}$ to $\mathrm{A}, \mathrm{BF}=27.4(\mathrm{ARMR}=0.35)$. All the other disseminations between production systems were also positive, but to a lesser degree than the ones mentioned above (Table 1). In addition, a sensitivity analysis aimed to evaluate the bias related to oversampling showed that while numerically it was a difference the interpretation remains the same, but we noticed that in the subsample there was even more support for the dissemination from company $\mathrm{B}$ to $\mathrm{C}$ and company $\mathrm{C}$ to $\mathrm{A}$ (Supplementary Table S7 for more details). Our analysis reveals that when comparing PRRSV dissemination between types of farms, the most frequent routes were linked with sow farms, especially when the direction of spread was from sow to nursery $(\mathrm{BF}>42)$. The highest support for this route occurred when we analyzed all systems combined $(\mathrm{BF}=109)$. The second most well-supported dissemination occurred from sow to finisher farms $(\mathrm{BF}>17.9)$, and as in the previous case, was best 
supported when we analyzed all systems together. $(\mathrm{BF}=80.5)$. Likewise, the dissemination between

nursery and finisher farms (in both directions) also showed positive support (BF $>6)$. However, all routes of dissemination that involved boar stud farms were rare events $(\mathrm{BF}<3)$ (Table 2). Furthermore, the sensitivity analysis showed similar trends, where the results of the equal sample sizes these were less sharp than the one using the original dataset. However, the transmissions that involved BoarStud were all negligible (Supplementary Table S8 for more details).

Within-production systems circulation (local) versus introduction of PRRSV from commercially

\section{unrelated farms (external)}

293 We tracked the origin of the migration events leading to the current PRRSV lineages circulating among the three pig systems, to determine whether detected PRRSV strains originated from within the same system (local) or from farms of non-commercially related pig systems (external introductions). For this analysis, we considered each coalescent event in the tree as a proxy for the timing of a transmission event and then classified each event as either a local transmission event if the parent and child lineages were both reconstructed to be in the local population or an external introduction if the parent lineage resided outside the local population. Overall, results showed a clear dominance of external transmission events that were observed in all pig companies; this tendency was constant during the whole period of study transmission events, while systems A and B exhibited 82.0\% in 2018 and $82.4 \%$ in 2017, respectively

307 higher during PRRSV season (mean=69.3) than the rest of the year (mean=60.1) $(\mathrm{Z}=-9.9, p<0.001)$.

\section{Factors promoting or restricting PRRSV dissemination direction}

309 To quantify the impact of each factor on PRRSV distribution, we calculated the association between

310 lineage duration and each covariate scaled distance through the $Q$ statistic method. We found strong 
311 support ( $Q$ value was positive) for pig density and distance to main roads treated as conductance factors,

312 while elevation and vegetation presented strong support as resistance factors (Supplementary Table S10

313 and Supplementary Figs. S5 and S6 for more details). These results also received statistical support

314 provided by Bayes factors (BF) since we observed that farms located in areas with a high density of pig

315 farms were more exposed to the introduction of PRRSV $(\mathrm{BF}>12)$. Likewise, the distance between the

316 farm and the nearest major road also showed a positive association with PRRSV diffusion (BF $>7)$. In

317 contrast, the factors identified as negative drivers, variables that act as barriers for the spread of PRRSV,

318 were higher elevation and denser vegetation $(\mathrm{BF}>3)$ (Supplementary Table S10). With respect to the analysis of the variables with significantly supported as conductance, analyzed as ranges (Supplementary

Table S11), the density of pigs ranging from 501-1.000 number of pigs $/ \mathrm{km}^{2}$ was sufficient to facilitate the spread of PRRSV when all sequences were analyzed together and for system A, while farms at B and C were more likely infected when pig density was above $1.000 \mathrm{pigs} / \mathrm{km}^{2}$. Likewise, farms located at a distance range of $1 \mathrm{~km}$ to $2 \mathrm{~km}$ of main roads showed to favor PRRSV introductions, while when systems a resistance range (Supplementary Table S11).

\section{Discussion}

331 This analysis detailed the evolutionary epidemiology of PRRSV among commercially related and

332 unrelated farms through continuous and discrete phylogeographic analyses. Overall, a decline in PRRSV

333 genetic diversity was observed over time. Such findings could have different explanations, one being a

334 rapid and efficient disseminating strain that was introduced into sow farms which consequently was

335 transferred to growing pig sites through regular pig movement. Another potential explanation could be

336 related to the efficacy of current interventions that local production systems have implemented, which 
reduced the volume and frequency of newly emerging strains, suggesting a more optimistic scenario than

proposed in a similar study done in another US region (Alkhamis et al., 2017). Given the economic impact and herd health relevance of new PRRSV strain introductions, it is imperative to evaluate how often PRRSV spreads among pig production systems, determine the role of each farm type, and identify

341 factors that may facilitate or keep PRRSV from spreading. We demonstrated that PRRSV circulation has

342 been mostly originated by transmission from outside of each pig production system, and this tendency is more predominant during the PRRSV season (from November until May) (MSHMP, 2020). understood which transmission mechanism plays a role in the dissemination across systems. Airborne spread has been suggested as a potential route (Dee et al., 2009; Otake et al., 2010). However, Arruda et al., $(2018,2019)$ did not reach that same conclusion. Pig farm density as expected arises as a risk factor in several studies and in our study, our phylodynamic findings agree with a regional spread as an important number of sequences does not directly represent all PRRSV cases circulating in this study region. There was important asymmetry in the number of sequences from boar studs, thus results of this farm type

356 should be interpreted with caution.

\section{$357 \quad$ PRRSV spatiotemporal dynamics}

358 The spatiotemporal dynamic pattern exhibited by PRRSV is the result of its transmission dynamics and

359 how circulating strains have been affected by viral selection (Pybus and Rambaut, 2009; Volz et al.,

360 2013). This can be observed in the shape of PRRSV phylodynamic patterns over the course of this study,

361 which shows a clear directional selection, which can result in the loss of genetic variation (Volz et al., 362 2013). Based on MSHMP data (MSHMP, 2020), vaccine usage increased between 2012 and 2014, which 
could have impacted PRRSV genetic diversity for system A after 2012. But the increment in the number of farms using vaccines during 2014 did not show a noticeable effect on PRRSV genetic variation. However, the increment in the application of MLV's in mid-2015 showed an undeniable positive effect on decreasing PRRSV genetic diversity (as seen in Figs. 2-5), similarly to the observations made by other authors (Sun et al., 2012; Jeong et al., 2018). However, to increase the robustness of future studies it is necessary to increase the farm coverage as well as the timeframe, not only related to the PRRSV genetic information but also to the vaccination data. than previous observations in a study that used data from one production system in another U.S. region

372 (34 km/year) (Alkhamis et al., 2017). This difference may be related to pig farm densities, being our 373 study region more populated with an average of 3 farms per $5 \mathrm{~km}^{2}$, and a more loyal representation of

374 PRRSV dynamics since we compiled samples of more than $92 \%$ of all farms in our study area..

375 Consequently, there are important aspects that have to be considered while analyzing the speed at which

376 PRRSV spreads, for example, those related with the overall pig health (i.e., absence of coinfections) and

377 factors related with on-farm biosecurity (i.e., presence of a line of separation, cleaning, and disinfection

378 station) (Rappole et al., 2006; Altizer et al., 2011; Lycett et al., 2019). Thus, further studies are needed to

379 evaluate how biosecurity practices and overall pig health may contribute in slowing or accelerating PRRSV spread, results of these assessments can be used to predict the most effective biosecurity measures and optimize control strategies that go beyond PRRSV (Silva et al., 2019), not to mention

382 current diseases that pose large-scale biosecurity threats to the U.S swine health such as African Swine

383 Fever.

384 PRRSV spread among and within farms of commercially unrelated production systems

385 Our analysis found relevant similarities in the spatiotemporal patterns of PRRSV spread among all farm 386 types of all production systems (Table 1,2), which was similar to previously reported findings that used 387 data from one production system in the U.S. (Alkhamis et al., 2016). At farm type levels, sow farms were by far the biggest spreader (Plain and Laurence, 2003) which was expected as they wean piglets on a 
weekly basis and these can be transported several miles away to an off-site growing pig facility.

Phylogenetic results also showed that PRRSV introductions into sow and nursery farms likely originated

from finisher farms, which could be partially related to gilt development units sending replacement gilts

to sow farms (Dee, 1995; Perez et al., 2015). Despite the substantial efforts to improve farms' biosecurity and immunization through the implementation of strategies such as, sow herd air filtration, gilt acclimation, virus elimination strategies (i.e., load-close-expose and rollover) which successfully reduced the number of PRRSV outbreaks (Corzo et al., 2010; Alonso et al., 2012; Velasova et al., 2012; Alonso et al., 2013; Blanchette, 2015; Silva et al., 2019), we continue to observe significant PRRSv dissemination by indirect routes (Corzo et al., 2010; Otake et al., 2010). Therefore, it is expected that changes in immunization protocols, such as varying vaccine brands at farms of one specific system may have a serious impact on PRRSV spread.

Within-production systems circulation (local) versus introduction of PRRSV from commercially

402 Between-farm animal movement has historically been associated with disease spread (Augusta et al., 2019; Moon et al., 2019; Machado et al., 2020). Here, we used structured coalescent models to infer by transmission between non-commercially related farms (external introductions) rather than within a production system (local). In addition, we observed that during PRRSV "season" (November-May), the external transmissions tended to be greater than during the rest of the year. While our analyses was not aimed to explore the route of transmission events or how viral lineages first entered a population

410 (Rasmussen et al., 2018), they reinforce the importance of PRRSV collateral spread and open the question 411 of how this dissemination is happening. Indeed, a recent work (Galvis et al., 2020) showed that for sow

412 farms more than $50 \%$ of the between-farm transmission occurred by proximity among infected and 413 susceptible farms, while $20 \%$ and $53 \%$ of the nurseries and finisher farms were infect by the same route, 414 respectively. While little is known about this collateral PRRSV propagations, at some level the external 
introduction have been associated with contaminated vehicles that service multiple production systems

416 (i.e., general maintenance, vaccination, cleaning or loading) (Otake et al., 2003; Dee et al., 2004), shared

417 equipment (Pitkin et al., 2009), non-pig animal vectors (Otake et al., 2002; Schurrer et al., 2005),

418 transport of semen (Christopher-Hennings et al., 1995) and airborne transmission (Lager and Mengeling,

419 2000; Trincado et al., 2004; Cho et al., 2007; Dee et al., 2009). At some degree, a contracted farm will

420 start producing pigs under a new production system, thus it is possible that a resident virus would be

421 introduced and become widespread within the new production system network. Knowledge gaps

422 regarding local dissemination of PRRSV still exist, hence, studies that consider phylodynamics coupled

423 with epidemiological models such as susceptible-infected-recovered to assess the relative contribution of

424 between-farm PRRSV transmission routes are needed. In addition, the proposed modeling should allowed

425 not only to answer "how it can spread" questions but also "what for", i.e. determining what effort is

426 required for reducing transmission (Beaunée et al., 2017).

427 Factors promoting or restricting PRRSV dissemination direction

428 In our PRRSV dissemination models, directions of lineages revealed the potential importance of two

429 factors that limited the spread of PRRSV. Vegetation density and high elevation, which are similar to

430 previous observations made by Mahesh et al., (2015), Arruda et al., (2017), and (Balka et al., 2018).

431 These authors also highlighted the important role that topography may play in mitigating the airborne

432 spread of PRRSV. In the same way, a study on foot-and-mouth disease, which also used continuous

433 phylogeographic analysis, identified that the presence of pure cropland or mixed land use both had a

434 negative impact on virus diffusion (Duchatel et al., 2019). Numerous studies have shown multiple

435 positive impacts of having vegetation barriers surrounding farms (i.e., trees, shrubs) over the airborne

436 spread of different pathogens (Malone, 2004; Patterson et al., 2008; Van Ryswyk et al., 2019), thus it

437 should be recognized as a possible biosecurity strategy. This study provided the first look at which

438 amount of healthy vegetation needed to protect farms from new introductions, consistently EVI index

439 between 41 and 45 showed to be sufficient. These values correspond to a dense tree coverage that is 
441 (Quercus virginiana), laurel oak (Quercus laurifolia) and loblolly pine (Pinus taeda) (Zhang et al., 2017).

442 Similarly, high elevated areas have proven to act as a natural topographic barrier for the local spread of

443 pathogens (Jacquot et al., 2017), where the slope of the terrain was found to have an association with a

444 lower PRRSV incidence (Arruda et al., 2017). We were able to identify a safe range of elevation that was

445 consistent above 61 meter from the sea level, while this can help the local production systems, other

446 regions of the US would need to be analyzed separately due to the differences possible elevation ranges.

447 The identification of the main drivers associated with PRRSV dissemination could help in deciding about

448 new farm locations, especially for systems located in highly dense pig areas (Yang et al., 2019). Farms

449 located in areas with higher farm density were more likely to sustain new PRRSV introductions, we highlight areas in which the volume of pigs by $\mathrm{km}^{2}$ above 500 animals, as has been previously described by Firkins and Weigel, (2004), Arruda et al., (2017) and Alkhamis et al., (2017, 2018). In addition, our results identified distance to the main road as associated with increased chances for PRRSV circulation.

This has led concern by the swine industry (Reicks, 2019), since vehicle movement has demonstrated to have a significant impact on increasing the transmission of several other diseases, for example, African Swine Fever (ASF) (Mur et al., 2012), Classical Swine Fever (CSF) (Bronsvoort et al., 2008), and for Foot and Mouth Disease (Muroga et al., 2013). Our findings indicate that farms located at $0.5 \mathrm{~km}$ to 0.7

$457 \mathrm{~km}$ from major roads were more easily exposed to PRRSV, therefore this distance could be used to make decisions about routes used for pig movement, the enhancement of on-farm biosecurity of sites within this range of distances to major roads, and in future expansions of operations new buildings could be built beyond $0.7 \mathrm{~km}$ from the main road.

462 describe and analyze the dissemination of PRRSV spread. Although the outcomes of phylodynamic 463 analyses can be exploited to investigate the impact of relevant factors on lineage dissemination diffusion,

464 it is important to note that such analyses depend on inferred viral lineage movement and thus, on the 465 spatial distribution of the sampled sequences (Dellicour et al., 2019). Our samples represent the 466 circulating PRRS viral population, as data comes from $\sim 92 \%$ of pig farms in one highly dense pig region, 
therefore can be directly applied in the region. Given that most commercial pig operation in North

468

469

470

471

472

473

474

475

476

477

478

479

480

481

482

483

484

485

486

487

488

489

490

491

492

America are similar but not the same our finding could also help the swine industry decision making on other parts of the county, conditional to data availability this analysis can be replicated at each relevant region or production system.

Our study was subject to a variety of limitations. The results of distance to main roads as a proxy for the exposure to PRRSV was inconsistent thus need to be interpreted with caution, when we analyzed all production systems together the distance of $1 \mathrm{~km}$ to $2 \mathrm{~km}$ was the only dangerous range, even though when analysis of individual systems showed that $0.7 \mathrm{~km}$ was enough this result need to the interpreted with caution. In the same why swine densities above $>1000$ pigs $/ \mathrm{km}^{2}$ were not supported as conductance while range of 500- $1000 \mathrm{pigs} / \mathrm{km}^{2}$, in both cases distance to main road and swine density, one reason for both inconsistence results could be related to the number of farm in such range intervals. For that reason, there is an urgent need to expand the current phylodynamic framework to allow for the calculation of point estimates of the likelihood of PRRSV spread driven by one and more predictor variables more directly, which would also considered categorical variables allowing us to determine relative safe or dangerous distances to main roads, for example (Dellicour et al., 2020).

\section{Conclusion}

Our study revealed significant asymmetries in the phylodynamic patterns among farms of three pig production systems. As a result of interventions that could be related to immunization and on-farm biosecurity, PRRSV genetic diversity has consistently declined in this region, especially in the last two years, which could explain why 2019 has had one of the lowest counts of PRRSV cases during its typical high season in the US (Sanhueza et al., 2019). Our results evidenced that PRRSV transmission across each system is most likely to originate from outside production systems. These results reiterate the importance of PRRSV farm proximity as a relevant route for virus dissemination. We highlight characteristics of locations to which PRRSV is more easily dispersed: highly dense pig population and proximity to main roads. On the protective side, higher elevation and more vegetation would be preferred. Thus, reinforcement of biosecurity may be the best tool. However, it raises the question of how much and 
which biosecurity practices have relevant impact on PRRSV spread. Answering this question requires

494 further studies capable of quantifying how biosecurity practices impede or reduce PRRSV dissemination.

\section{Acknowledgements}

497 We acknowledge the Department of Population Health and Pathobiology: North Carolina State University 498 provided startup funds for Dr. Machado and CVM for the intramural grant which supported Dr. Jara. This

499 work was also supported by Critical Agricultural Research and Extension 2019-68008-29910 from the

500 USDA National Institute of Food and Agriculture. The Morrison Swine Health Monitoring Project is a

501 Swine Health Information Center funded project. Authors would like to acknowledge participating

502 systems and veterinarians.

503 Authors' contributions

504 MJ and GM conceived the paper ideas. MJ, DR, and GM participated in the design of the study. CC

505 coordinated the PRRSV data collection. MJ conducted data processing and cleaning. MJ performed the 506 phylodynamic analysis. MJ, DR, CAC, and GM wrote and edited the manuscript. All authors discussed

507 the results and critically reviewed the manuscript.

508 Conflict of interest

509 All authors confirm that there are no conflicts of interest to declare

$510 \quad$ Ethical statement

511 The authors confirm the ethical policies of the journal, as noted on the journal's author guidelines page.

512 Since this work did not involve animal sampling nor questionnaire data collection by the researchers there

513 was no need for ethics permits.

\section{Data Availability Statement}

515 The data that support the findings of this study are not publicly available and are protected by confidential 516 agreements, therefore, are not available.

\section{$518 \quad$ References}


Alkhamis, M., A. Perez, M. Murtaugh, X. Wang, and R. Morrison, 2016: Applications of Bayesian phylodynamic methods in a recent U.S. porcine reproductive and respiratory syndrome virus outbreak. Front. Microbiol. 7, DOI: 10.3389/fmicb.2016.00067.

Alkhamis, M.A., A.G. Arruda, R.B. Morrison, and A.M. Perez, 2017: Novel approaches for Spatial and Molecular Surveillance of Porcine Reproductive and Respiratory Syndrome Virus (PRRSv) in the United States. Sci. Rep. 7, DOI: 10.1038/s41598-017-04628-2.

Alkhamis, M.A., A.G. Arruda, C. Vilalta, R.B. Morrison, and A.M. Perez, 2018: Surveillance of porcine reproductive and respiratory syndrome virus in the United States using risk mapping and species distribution modeling. Prev. Vet. Med. 150, 135-142, DOI: 10.1016/j.prevetmed.2017.11.011.

Alonso, C., M.P. Murtaugh, S.A. Dee, and P.R. Davies, 2013: Epidemiological study of air filtration systems for preventing PRRSV infection in large sow herds. Prev. Vet. Med. 112, 109-117, DOI: 10.1016/j.prevetmed.2013.06.001.

Alonso, C., S. Otake, P. Davies, and S. Dee, 2012: An evaluation of interventions for reducing the risk of PRRSV introduction to filtered farms via retrograde air movement through idle fans. Vet. Microbiol. 157, 304-310, DOI: 10.1016/j.vetmic.2012.01.010.

Altizer, S., R. Bartel, and B.A. Han, 2011: Animal migration and infectious disease risk. Science (80-. ). 331, 296-302, DOI: 10.1126/science.1194694.

Arruda, A.G., J. Sanhueza, C. Corzo, and C. Vilalta, 2018: Assessment of area spread of porcine reproductive and respiratory syndrome (PRRS) virus in three clusters of swine farms. Transbound. Emerg. Dis. 65, 1282-1289, DOI: 10.1111/tbed.12875.

Arruda, A.G., S. Tousignant, J. Sanhueza, C. Vilalta, Z. Poljak, M. Torremorell, C. Alonso, and C.A. Corzo, 2019: Aerosol Detection and Transmission of Porcine Reproductive and Respiratory Syndrome Virus (PRRSV): What Is the Evidence, and What Are the Knowledge Gaps? Viruses 11, 712, DOI: $10.3390 / \mathrm{v} 11080712$.

Arruda, A.G., C. Vilalta, A. Perez, and R. Morrison, 2017: Land altitude, slope, and coverage as risk factors for Porcine Reproductive and Respiratory Syndrome (PRRS) outbreaks in the United States. 
PLoS One 12, DOI: 10.1371/journal.pone.0172638.

546

547

548

549

550

551

552

553

554

555

556

557

558

559

560

561

562

563

564

565

566

567
Augusta, C., G.W. Taylor, and R. Deardon, 2019: Dynamic contact networks of swine movement in Manitoba, Canada: Characterization and implications for infectious disease spread. Transbound. Emerg. Dis. 66, 1910-1919, DOI: 10.1111/tbed.13220.

Baele, G., S. Dellicour, M.A. Suchard, P. Lemey, and B. Vrancken, 2018: Recent advances in computational phylodynamics. Curr. Opin. Virol. 31, 24-32, DOI: 10.1016/J.COVIRO.2018.08.009.

Balka, G., K. Podgórska, M.S. Brar, Á. Bálint, D. Cadar, V. Celer, L. Dénes, Z. Dirbakova, A. Jedryczko, L. Márton, D. Novosel, T. Petrović, I. Sirakov, D. Szalay, I. Toplak, F.C.C. Leung, and T. Stadejek, 2018: Genetic diversity of PRRSV 1 in Central Eastern Europe in 1994-2014: Origin and evolution of the virus in the region. Sci. Rep. 8, 1-12, DOI: 10.1038/s41598-018-26036-w.

Beaunée, G., E. Vergu, A. Joly, and P. Ezanno, 2017: Controlling bovine paratuberculosis at a regional scale: Towards a decision modelling tool. J. Theor. Biol. 435, 157-183, DOI: 10.1016/j.jtbi.2017.09.012.

Bielejec, F., G. Baele, B. Vrancken, M. Suchard, A. Rambaut, and P. Lemey, 2016: SpreaD3: interactive visualization of spatiotemporal history and trait evolutionary processes. Mol. Biol. Evol. 33, 21672169.

Blanchette, A., 2015: Herding species: Biosecurity, posthuman labor, and the american industrial pig. Cult. Anthropol. 30, 640-669, DOI: 10.14506/ca30.4.09.

Bouckaert, R., J. Heled, D. Kühnert, T. Vaughan, C.H. Wu, D. Xie, M.A. Suchard, A. Rambaut, and A.J. Drummond, 2014: BEAST 2: A Software Platform for Bayesian Evolutionary Analysis. (Andreas Prlic, Ed.)PLoS Comput. Biol. 10, e1003537, DOI: 10.1371/journal.pcbi.1003537.

Bronsvoort, B.M.D.C., S. Parida, I. Handel, S. McFarland, L. Fleming, P. Hamblin, and R. Kock, 2008: Serological survey for foot-and-mouth disease virus in wildlife in eastern Africa and estimation of test parameters of a nonstructural protein enzyme-linked immunosorbent assay for buffalo. Clin.

Vaccine Immunol. 15, 1003-11, DOI: 10.1128/CVI.00409-07. 
571 Cavanagh, D., D.A. Brian, L. Enjuanes, K. V. Holmes, M.M.C. Lai, H. Laude, S.G. Siddell, W. Spaan, F.

572 Taguchi, and P.J. Talbot, 1990: Recommendations of the coronavirus study group for the nomenclature of the structural proteins, mRNAs, and genes of coronaviruses. Virology 176, 306307, DOI: 10.1016/0042-6822(90)90259-T.

Cho, J.G., J. Deen, and S.A. Dee, 2007: Influence of isolate pathogenicity on the aerosol transmission of

\section{7}

578

579

580

581

582

583

584

585

586

587

588

589

590

591

Porcine reproductive and respiratory syndrome virus. Can. J. Vet. Res. 71, $23-27$.

Christopher-Hennings, J, E.A. Nelson, R.J. Hines, J.K. Nelson, S.L. Swenson, J.J. Zimmerman, C.L. Chase, M.J. Yaeger, and D.A. Benfield, 1995: Persistence of porcine reproductive and respiratory syndrome virus in serum and semen of adult boars. J. Vet. Diagn. Invest. 7, 456-64, DOI: $10.1177 / 104063879500700406$.

Christopher-Hennings, Jane, 2001: Monitoring for porcine reproductive and respiratory syndrome virus (PRRSV) in the boar stud. J. Swine Heal. Prod. 9, 186-188.

Corzo, C.A., E. Mondaca, S. Wayne, M. Torremorell, S. Dee, P. Davies, and R.B. Morrison, 2010: Control and elimination of porcine reproductive and respiratory syndrome virus. Virus Res. 154, 185-192, DOI: 10.1016/j.virusres.2010.08.016.

De Smit, A.J., A. Bouma, C. Terpstra, and J.T. Van Oirschot, 1999: Transmission of classical swine fever virus by artificial insemination. Vet. Microbiol. 67, 239-249, DOI: 10.1016/S0378-1135(99)000450.

Dee, S. A., 1995: Controlling the spread of PRRS virus in the breeding herd through management of the gilt pool. Swine Heal. Prod. 3, 64-69.

Dee, S. A., S. Otake, S. Oliveira, and J. Deen, 2009: Evidence of long distance airborne transport of porcine reproductive and respiratory syndrome virus and Mycoplasma hyopneumoniae. Vet. Res. 40, 39, DOI: $10.1051 /$ vetres/2009022.

Dee, Scott A., J. Deen, and C. Pijoan, 2004: Evaluation of 4 intervention strategies to prevent the mechanical transmission of porcine reproductive and respiratory syndrome virus. Can. J. Vet. Res.
68, 19-26. 
Dellicour, S., D. Desmecht, J. Paternostre, C. Malengreaux, A. Licoppe, M. Gilbert, and A. Linden, 2020: Unravelling the dispersal dynamics and ecological drivers of the African swine fever outbreak in Belgium. (Andrew Park, Ed.)J. Appl. Ecol.1365-2664.13649, DOI: 10.1111/1365-2664.13649.

Dellicour, S., P. Lemey, J. Artois, T.T. Lam, A. Fusaro, I. Monne, G. Cattoli, D. Kuznetsov, I. Xenarios, G. Dauphin, W. Kalpravidh, S. Von Dobschuetz, F. Claes, S.H. Newman, M.A. Suchard, G. Baele, and M. Gilbert, 2019: Incorporating heterogeneous sampling probabilities in continuous phylogeographic inference - Application to H5N1 spread in the Mekong region. Bioinformatics1-7, DOI: 10.1093/bioinformatics/btz882.

Dellicour, S., R. Rose, N.R. Faria, P. Lemey, and O.G. Pybus, 2016: SERAPHIM: Studying environmental rasters and phylogenetically informed movements. Bioinformatics 32, 3204-3206, DOI: 10.1093/bioinformatics/btw384.

Dellicour, S., R. Rose, and O.G. Pybus, 2016: Explaining the geographic spread of emerging epidemics: a framework for comparing viral phylogenies and environmental landscape data. BMC Bioinformatics 17, 82, DOI: 10.1186/s12859-016-0924-x.

Dellicour, S., C. Troupin, F. Jahanbakhsh, A. Salama, S. Massoudi, M.K. Moghaddam, G. Baele, P. Lemey, A. Gholami, and H. Bourhy, 2019: Using phylogeographic approaches to analyse the dispersal history, velocity and direction of viral lineages - Application to rabies virus spread in Iran. Mol. Ecol. 28, 4335-4350, DOI: 10.1111/mec.15222.

Dokland, T., 2010: The structural biology of PRRSV. Virus Res. 154, 86-97, DOI: 10.1016/j.virusres.2010.07.029.

Drummond, A.J., S.Y.W. Ho, M.J. Phillips, and A. Rambaut, 2006: Relaxed phylogenetics and dating with confidence. (David Penny, Ed.)PLoS Biol. 4, e88, DOI: 10.1371/journal.pbio.0040088.

Duchatel, F., M. Bronsvoort, and S. Lycett, 2019: Phylogeographic analysis and identification of factors impacting the diffusion of Foot-and-Mouth disease virus in Africa. Front. Ecol. Evol. 7, 371.

Dudas, G., L.M. Carvalho, T. Bedford, A.J. Tatem, G. Baele, N.R. Faria, and F. Bielejec, 2017: Virus genomes reveal factors that spread and sustained the Ebola epidemic. Nature 544, 309-315, DOI: 
Faria, N.R., N. Vidal, J. Lourenco, J. Raghwani, K.C.E. Sigaloff, A.J. Tatem, D.A.M. Van De Vijver,

625

626

627

628

629

630

631

632

633

634

635

636

637

638

639

640

641

642

643

644

645

646

647

648

A.C. Pineda-Peña, R. Rose, C.L. Wallis, S. Ahuka-Mundeke, J.J. Muyembe-Tamfum, J. Muwonga, M.A. Suchard, T.F.R. De Wit, R.L. Hamers, N. Ndembi, G. Baele, M. Peeters, O.G. Pybus, P. Lemey, and S. Dellicour, 2019: Distinct rates and patterns of spread of the major HIV-1 subtypes in Central and East Africa. PLoS Pathog. 15, e1007976-e1007976, DOI:

10.1371/journal.ppat.1007976.

Firkins, L.D., and R.M. Weigel, 2004: A retrospective study of risk factors for porcine reproductive and respiratory syndrome virus infection and clinical disease in swine herds in Illinois during the early years of the pandemic. J. Swine Heal. Prod. 12, 23-28.

Fountain-Jones, N.M., W.D. Pearse, L.E. Escobar, A. Alba-Casals, S. Carver, T.J. Davies, S. Kraberger, M. Papeş, K. Vandegrift, K. Worsley-Tonks, and M.E. Craft, 2018: Towards an eco-phylogenetic framework for infectious disease ecology. Biol. Rev. 93, 950-970, DOI: 10.1111/brv.12380.

Franzo, G., G. Dotto, M. Cecchinato, D. Pasotto, M. Martini, and M. Drigo, 2015: Phylodynamic analysis of porcine reproductive and respiratory syndrome virus (PRRSV) in Italy: Action of selective pressures and interactions between different clades. Infect. Genet. Evol. 31, 149-157, DOI: 10.1016/j.meegid.2015.01.026.

Galvis, J.A., J. Prada, C. Jones, and G. Machado, 2020: Transmission and control strategies for porcine reproductive and respiratory syndrome virus. bioRxiv2020.05.23.112946, DOI: 10.1101/2020.05.23.112946.

Gill, M.S., P. Lemey, S.N. Bennett, R. Biek, and M.A. Suchard, 2016: Understanding Past Population Dynamics: Bayesian Coalescent-Based Modeling with Covariates. Syst. Biol. 65, 1041-1056, DOI: 10.1093/sysbio/syw050.

Guindon, S., J.F. Dufayard, V. Lefort, M. Anisimova, W. Hordijk, and O. Gascuel, 2010: New algorithms and methods to estimate maximum-likelihood phylogenies: Assessing the performance of PhyML 3.0. Syst. Biol. 59, 307-321, DOI: 10.1093/sysbio/syq010. 
Hasegawa, M., H. Kishino, and T. Yano, 1985: Dating of the human-ape splitting by a molecular clock of mitochondrial DNA. J. Mol. Evol. 22, 160-174, DOI: 10.1007/BF02101694.

Hicks, J.T., D.H. Lee, V.R. Duvuuri, M.K. Torchetti, D.E. Swayne, and J. Bahl, 2020: Agricultural and geographic factors shaped the North American 2015 highly pathogenic avian influenza H5N2 outbreak. PLoS Pathog. 16, e1007857, DOI: 10.1371/journal.ppat.1007857.

Hill, V., and G. Baele, 2019: Bayesian estimation of past population dynamics in BEAST 1.10 using the Skygrid coalescent model. Mol. Biol. Evol. 36, 2620-2628.

Hogg, R. V, E.A. Tanis, D.L. Zimmerman, B. Columbus, I. New, Y. San, F. Upper, S. River, A. Cape, T. Dubai, L. Madrid, M. Munich, P. Montreal, T. Delhi, M.C. São, P. Sydney, H. Kong, S. Singapore, and T. Tokyo, 2010: Probability and Statistical Inference. Pearson/Prentice Hall.

Holtkamp, D.J., J.B. Kliebenstein, E. Neumann, J.J. Zimmerman, H. Rotto, T.K. Yoder, C. Wang, P. Yeske, C.L. Mowrer, C.A. Haley, E.J. Neumann, H.F. Rotto, and P.E. Yeske, 2013: Assessment of the economic impact of porcine reproductive and respiratory syndrome virus on United States pork producers Assessment of the economic impact of porcine reproductive and respiratory syndrome virus on United States pork producers Assessment of. J. Swine Heal. Prod. 21.

Jacquot, M., K. Nomikou, M. Palmarini, P. Mertens, and R. Biek, 2017: Bluetongue virus spread in Europe is a consequence of climatic, landscape and vertebrate host factors as revealed by phylogeographic inference. Proc. R. Soc. B Biol. Sci. 284, DOI: 10.1098/rspb.2017.0919.

Jeong, J., S. Kim, C. Park, I. Kang, K.H. Park, H.J. Ham, and C. Chae, 2018: Effect of vaccination with a porcine reproductive and respiratory syndrome subunit vaccine on sow reproductive performance in endemic farms. Vet. Rec. 182, 602, DOI: 10.1136/vr.104547.

Kalyaanamoorthy, S., B.Q. Minh, T.K.F. Wong, A. Von Haeseler, and L.S. Jermiin, 2017: ModelFinder: Fast model selection for accurate phylogenetic estimates. Nat. Methods 14, 587-589, DOI: 10.1038/nmeth.4285.

Kass, R.E., and A.E. Raftery, 1995: Bayes Factors. J. Am. Stat. Assoc. 90, 773-795, DOI: 10.1080/01621459.1995.10476572. 
675

676

677

678

679

680

681

682

683

684

685

686

687

688

689

690

691

692

693

694

695

696

697

698

699

700

Kumar, S., M. Nei, J. Dudley, and K. Tamura, 2008: MEGA: A biologist-centric software for evolutionary analysis of DNA and protein sequences. Brief. Bioinform. 9, 299-306, DOI: 10.1093/bib/bbn017.

Kumar, Sudhir, G. Stecher, M. Li, C. Knyaz, and K. Tamura, 2018: MEGA X: Molecular Evolutionary Genetics Analysis across Computing Platforms | Molecular Biology and Evolution | Oxford Academic. Mol. Biol. Evol. 35, 1547-1549.

Laenen, L., S. Dellicour, V. Vergote, I. Nauwelaers, S. De Coster, I. Verbeeck, B. Vanmechelen, P. Lemey, and P. Maes, 2016: Spatio-temporal analysis of Nova virus, a divergent hantavirus circulating in the European mole in Belgium. Mol. Ecol. 25, 5994-6008, DOI: 10.1111/mec.13887.

Lager, K.M., and W.L. Mengeling, 2000: Experimental aerosol transmission of pseudorabies virus and porcine reproductive and respiratory syndrome virus. Proc. Am. Assoc. Swine Pract.409-410.

Lemey, P., A. Rambaut, A.J. Drummond, and M.A. Suchard, 2009: Bayesian Phylogeography Finds Its Roots. (Christophe Fraser, Ed.)PLoS Comput. Biol. 5, e1000520, DOI: 10.1371/journal.pcbi.1000520.

Linhares, D.C.L., J.P. Cano, M. Torremorell, and R.B. Morrison, 2014: Comparison of time to PRRSvstability and production losses between two exposure programs to control PRRSv in sow herds. Prev. Vet. Med. 116, 111-119, DOI: 10.1016/j.prevetmed.2014.05.010.

Lycett, S.J., V.N. Tanya, M. Hall, D. King, S. Mazeri, V. Mioulet, N. Knowles, J. Wadsworth, K. Bachanek-Bankowska, N.N. Victor, K.L. Morgan, and M. Bronsvoort, 2019: The evolution and phylodynamics of serotype A and SAT2 foot-and-mouth disease viruses in endemic regions of Africa. Sci. Rep. 9, 5614, DOI: 10.1101/572198.

Machado, G., J.A. Galvis, F.P.N. Lopes, J. Voges, A.A.R. Medeiros, and N.C. Cárdenas, 2020:

Quantifying the dynamics of pig movements improves targeted disease surveillance and control plans. bioRxiv2019.12.29.886408, DOI: 10.1101/2019.12.29.886408.

Mahesh, K.C., J. Bhoj Raj, S. Swoyam, Prakash, M. Prajapati, D. Kathayat, and S. Dhakal, 2015: SeroPrevalence of Porcine Reproductive and Respiratory Syndrome (PRRS) in Pigs of Different 

10.3126/ijasbt.v3i2.12539.

Malone, B., 2004: Using trees to reduce dust and odor emissions from poultry farmsPoult. Inf. Exch.

Martin, D.P., B. Murrell, M. Golden, A. Khoosal, and B. Muhire, 2015: RDP4: Detection and analysis of recombination patterns in virus genomes. Virus Evol. 1, DOI: 10.1093/ve/vev003.

Moon, S.A., T. Ferdousi, A. Self, and C.M. Scoglio, 2019: Estimation of swine movement network at farm level in the US from the Census of Agriculture data. Sci. Rep. 9, DOI: 10.1038/s41598-019-

MSHMP, 2020: PRRS charts [Online] Available at https://www.vetmed.umn.edu/centersprograms/swine-program/outreach-leman-mshmp/mshmp/mshmp-prrs-figures (accessed January 10,

Müller, N.F., D. Rasmussen, and T. Stadler, 2018: MASCOT: Parameter and state inference under the marginal structured coalescent approximation. Bioinformatics 34, 3843-3848, DOI: 10.1093/bioinformatics/bty406.

Müller, N.F., D.A. Rasmussen, and T. Stadler, 2017: The structured coalescent and its approximations. Mol. Biol. Evol. 34, 2970-2981, DOI: 10.1093/molbev/msx186.

Mur, L., B. Martínez-López, and J.M. Sánchez-Vizcaíno, 2012: Risk of African swine fever introduction into the European Union through transport-associated routes: returning trucks and waste from international ships and planes. BMC Vet. Res. 8, 149, DOI: 10.1186/1746-6148-8-149.

Muroga, N., S. Kobayashi, T. Nishida, Y. Hayama, T. Kawano, T. Yamamoto, and T. Tsutsui, 2013: Risk factors for the transmission of foot-and-mouth disease during the 2010 outbreak in Japan: a case-control study. BMC Vet. Res. 9, 150, DOI: 10.1186/1746-6148-9-150.

Murray, G.G.R., F. Wang, E.M. Harrison, G.K. Paterson, A.E. Mather, S.R. Harris, M.A. Holmes, A. Rambaut, and J.J. Welch, 2016: The effect of genetic structure on molecular dating and tests for temporal signal. Methods Ecol. Evol. 7, 80-89, DOI: 10.1111/2041-210X.12466.

726 Navascués, M., F. Depaulis, and B. Emerson, 2010: Combining contemporary and ancient DNA in 
population genetic and phylogeographical studies. Mol. Ecol. Resour. 10, 760-772, DOI:

$$
\text { 10.1111/j.1755-0998.2010.02895.x. }
$$

Neumann, E.J., J.B. Kliebenstein, C.D. Johnson, J.W. Mabry, E.J. Bush, A.H. Seitzinger, A.L. Green, and J.J. Zimmerman, 2005: Assessment of the economic impact of porcine reproductive and respiratory syndrome on swine production in the United States. J. Am. Vet. Med. Assoc. 227, 385-392, DOI: 10.2460/javma.2005.227.385.

Nguyen, L.T., H.A. Schmidt, A. Von Haeseler, and B.Q. Minh, 2015: IQ-TREE: A fast and effective stochastic algorithm for estimating maximum-likelihood phylogenies. Mol. Biol. Evol. 32, 268-274, DOI: $10.1093 / \mathrm{molbev} / \mathrm{msu300.}$

Otake, S., S. Dee, K. Rossow, R. Moon, and C. Pijoan, 2002: Mechanical transmission of porcine reproductive and respiratory syndrome virus by mosquitoes, Aedes vexans (Meigen). Can. J. Vet. Res. 66, 191-195.

Otake, Satoshi., S. Dee, C. Corzo, S. Oliveira, and J. Deen, 2010: Long-distance airborne transport of infectious PRRSV and Mycoplasma hyopneumoniae from a swine population infected with multiple viral variants. Vet. Microbiol. 145, 198-208, DOI: 10.1016/j.vetmic.2010.03.028.

Otake, Satoshi, S.. Otake, S., R.D. Dee, K.D. Moon, C. Roscow, 265-270. Trincado Pijoan, 2003: Evaluation of mosquitoes, Aedes vexans, as biological vectors of porcine reproductive and respiratory syndrome virus. Can. J. Vet. Res. 67, S.A. Dee, R.D. Moon, K.D. Roscow, C. Trincado, and C. Pijoan, 2003: Evaluation of mosquitoes, Aedes vexans, as biological vectors of porcine reproductive and respiratory syndrome virus. Can. J. Vet. Res. 67, 265-270.

Passafaro, T.L., A.F.A. Fernandes, B.D. Valente, N.H. Williams, and G.J.M. Rosa, 2020: Network analysis of swine movements in a multi-site pig production system in Iowa, USA. Prev. Vet. Med. 174, DOI: 10.1016/j.prevetmed.2019.104856.

Patterson, P.H., A. Adrizal, R.M. Hulet, R.M. Bates, C.A.B. Myers, G.P. Martin, R.L. Shockey, M. Van Der Grinten, D.A. Anderson, and J.R. Thompson, 2008: Vegetative buffers for fan emissions from poultry farms: 2. Ammonia, dust and foliar nitrogen. J. Environ. Sci. Heal. - Part B Pestic. Food 
Contam. Agric. Wastes 43, 96-103, DOI: 10.1080/03601230701735078.

Perez, A.M., P.R. Davies, C.K. Goodell, D.J. Holtkamp, E. Mondaca-Fernández, Z. Poljak, S.J. Tousignant, P. Valdes-Donoso, J.J. Zimmerman, and R.B. Morrison, 2015: Lessons learned and knowledge gaps about the epidemiology and control of porcine reproductive and respiratory syndrome virus in North America. J. Am. Vet. Med. Assoc. 246, 1304-1317, DOI: 10.2460/javma.246.12.1304.

Pileri, E., and E. Mateu, 2016: Review on the transmission porcine reproductive and respiratory syndrome virus between pigs and farms and impact on vaccination. Vet. Res. 47, 1-13, DOI: 10.1186/s13567016-0391-4.

Pitkin, A., J. Deen, and S. Dee, 2009: Use of a production region model to assess the airborne spread of porcine reproductive and respiratory syndrome virus. Vet. Microbiol. 136, 1-7, DOI: 10.1016/j.vetmic.2008.10.013.

Plain, R.L., and J.D. Laurence, 2003: Swine production. Vet. Clin. North Am. Food Anim. Pract. 19, 319 337.

Pybus, O., M. Suchard, P. Lemey, F. Bernardin, A. Rambaut, F. Crawford, R. Gray, N. Arinaminpathy, S. Stramer, M. Busch, and E. Delwart, 2012: Unifying the spatial epidemiology and molecular evolution of emerging epidemics. Natl. Acad Sci.1-6, DOI: 10.1073/pnas.1206598109//DCSupplemental.

Pybus, O.G., and A. Rambaut, 2009: Evolutionary analysis of the dynamics of viral infectious disease. Nat. Rev. Genet. 10, 540-550, DOI: 10.1038/nrg2583.

Rambaut, A., and A. Drummond, 2016: TreeAnnotator v.2.4. 3. . Part of the BEAST package.

Rambaut, A., T. Lam, L. Max Carvalho, and O. Pybus, 2016: Exploring the temporal structure of heterochronous sequences using TempEst (formerly Path-O-Gen). Virus Evol. 2, vew007, DOI: 10.1093/ve/vew007.

Rappole, J.H., B.W. Compton, P. Leimgruber, J. Robertson, D.I. King, and S.C. Renner, 2006: Modeling movement of West Nile virus in the Western Hemisphere. Vector-Borne Zoonotic Dis. 6, 128-139, 
DOI: $10.1089 /$ vbz.2006.6.128.

Rasmussen, D.A., E. Wilkinson, A. Vandormael, F. Tanser, D. Pillay, T. Stadler, and T. de Oliveira, 2018: Tracking external introductions of HIV using phylodynamics reveals a major source of infections in rural KwaZulu-Natal, South Africa. Virus Evol. 4, vey037, DOI: 10.1093/ve/vey037.

Rathkjen, P.H., and J. Dall, 2017: Control and eradication of porcine reproductive and respiratory syndrome virus type 2 using a modified-live type 2 vaccine in combination with a load, close, homogenise model: An area elimination study. Acta Vet. Scand. 59, 4, DOI: 10.1186/s13028-0160270-z.

Reicks, D.L., 2019: Effective biosecurity to protect North American studs and clients from emerging infectious disease. Theriogenology 137, 82-87, DOI: 10.1016/j.theriogenology.2019.05.041.

Sanhueza, J.M., C. Vilalta, M. Kikuti, E. Geary, and C. Corzo, 2019: Comparison of PRRS incidence rate in status IV breeding herds during 2009-2019.

Sanhueza, Juan M., C. Vilalta, C. Corzo, and A.G. Arruda, 2019: Factors affecting Porcine Reproductive and Respiratory Syndrome virus time-to-stability in breeding herds in the Midwestern United States. Transbound. Emerg. Dis. 66, 823-830, DOI: 10.1111/tbed.13091.

Schurrer, J., S. Dee, R. Moon, M. Murthaugh, C. Finnegan, J. Deen, S. Kleiboeker, and C. Pijoan, 2005: Retention of ingested porcine reproductive and respiratory syndrome virus in houseflies. Am Vet Med Assoc 66, 1517-1525, DOI: 10.2460/ajvr.2005.66.1517.

Shi, M., T. Tsan-Yuk Lam, C.-C. Hon, R. Kin-Hei Hui, K.S. Faaberg, T. Wennblom, M.P. Murtaugh, T. Stadejek, and F. Chi-Ching Leung, 2010: Molecular epidemiology of PRRSV: A phylogenetic perspective. Virus Res. 154, 7-17, DOI: 10.1016/j.virusres.2010.08.014.

Silva, G.S., G. Machado, K.L. Baker, D.J. Holtkamp, and D.C.L. Linhares, 2019: Machine-learning algorithms to identify key biosecurity practices and factors associated with breeding herds reporting PRRS outbreak. Prev. Vet. Med. 171, DOI: 10.1016/j.prevetmed.2019.104749.

Sørensen, R., U. Zinko, and J. Seibert, 2005: On the calculation of the topographic wetness index: evaluation of different methods based on field observations On the calculation of the topographic 
wetness index: evaluation of different methods based on field observations Evaluation of TWI calculation Vol. 2Hydrol. Earth Sys. Sci. Discuss.

Sun, R.Q., R.J. Cai, Y.Q. Chen, P.S. Liang, D.K. Chen, and C.X. Song, 2012: Outbreak of porcine epidemic diarrhea in suckling piglets, China. Emerg. Infect. Dis. 18, 161-163, DOI:

Sun, Y.K., X.L. Han, Y.F. Wei, Z.Q. Yu, C.H. Ji, Q. Li, G. Lu, L. Shen, C.Q. Ma, H. Wang, and G.H. Zhang, 2019: Phylogeography, phylodynamics and the recent outbreak of lineage 3 porcine reproductive and respiratory syndrome viruses in China. Transbound. Emerg. Dis. 66, 2152-2162,

Tavaré, S., 1986: Some probabilistic and statistical problems in the analysis of dna sequences. Lect Math Life Sci. 17, 57-86.

To, T.H., M. Jung, S. Lycett, and O. Gascuel, 2016: Fast Dating Using Least-Squares Criteria and Algorithms. Syst. Biol. 65, 82-97, DOI: 10.1093/sysbio/syv068. porcine reproductive and respiratory syndrome virus by aerosols under controlled field conditions. Vet. Rec. 154, 294-297, DOI: 10.1136/vr.154.10.294.

Trovão, N.S., M.A. Suchard, G. Baele, M. Gilbert, and P. Lemey, 2015: Bayesian inference reveals hostspecific contributions to the epidemic expansion of influenza A H5N1. Mol. Biol. Evol. 32, 32643275, DOI: 10.1093/molbev/msv185.

Van Ryswyk, K., N. Prince, M. Ahmed, E. Brisson, J.D. Miller, and P.J. Villeneuve, 2019: Does urban vegetation reduce temperature and air pollution concentrations? Findings from an environmental and respiratory syndrome virus infection and resulting challenges for effective disease surveillance. BMC Vet. Res. 8, 184, DOI: 10.1186/1746-6148-8-184. 
831 Volz, E.M., K. Koelle, and T. Bedford, 2013: Viral Phylodynamics. PLoS Comput. Biol. 9, DOI: 10.1371/journal.pcbi.1002947.

Yang, J., N.F. Müller, R. Bouckaert, B. Xu, and A.J. Drummond, 2019: Bayesian phylodynamics of avian influenza A virus H9N2 in Asia with time-dependent predictors of migration. PLoS Comput. Biol. 15, DOI: 10.1371/journal.pcbi.1007189.

Yang, Z., 1994: Maximum likelihood phylogenetic estimation from DNA sequences with variable rates over sites: Approximate methods. J. Mol. Evol. 39, 306-314, DOI: 10.1007/BF00160154.

Yuan, S., C.J. Nelsen, M.P. Murtaugh, B.J. Schmitt, and K.S. Faaberg, 1999: Recombination between North American strains of porcine reproductive and respiratory syndrome virus. Virus Res. 61, 87-

Zhang, X., S. Wu, X. Yan, and Z. Chen, 2017: A global classification of vegetation based on NDVI, rainfall and temperature. Int. J. Climatol. 37, 2318-2324, DOI: 10.1002/joc.4847.

Table list

Table 1. PRRSV dissemination between systems. Bayes factor results represent the level of support in the 846 rates of the spread between systems, where $\mathrm{BF}>20$ is strong support, $\mathrm{BF}=3-19$ is positive support and $847 \mathrm{BF}<3$ is negligible.

\begin{tabular}{ccccc}
\hline Origin & Destination & $\begin{array}{c}\text { Average relative migration } \\
\text { rate }\end{array}$ & $\begin{array}{c}\text { Bayes } \\
\text { factor }\end{array}$ & Interpretation \\
\hline A & B & $1.79 \pm 0.92$ & 102.1 & Strong support \\
A & C & $0.46 \pm 0.39$ & 47.8 & Strong support \\
B & A & $0.35 \pm 0.37$ & 27.4 & Strong support \\
B & C & $0.33 \pm 0.13$ & 7.9 & Positive support \\
C & A & $0.41 \pm 0.22$ & 14.7 & Positive support \\
C & B & $0.39 \pm 0.16$ & 10.3 & Positive support \\
\hline
\end{tabular}

Table 2. PRRSV dissemination between types of farms. Bayes factor results represent the level of support in the rates of the spread between types of farms, where $\mathrm{BF}>20$ is strong support, $\mathrm{BF}=3-19$ is positive 851 support and $\mathrm{BF}<3$ is negligible. 


\begin{tabular}{cccccc}
\hline $\begin{array}{c}\text { Farm type } \\
\text { of origin }\end{array}$ & $\begin{array}{c}\text { Farm type of } \\
\text { destination }\end{array}$ & $\begin{array}{c}\text { All systems } \\
\text { (Bayes factor) }\end{array}$ & $\begin{array}{c}\text { System A } \\
\text { (Bayes factor) }\end{array}$ & $\begin{array}{c}\text { System B } \\
\text { (Bayes factor) }\end{array}$ & $\begin{array}{c}\text { System C } \\
\text { (Bayes factor) }\end{array}$ \\
\hline Sow & Nursery & 109 & 56.2 & 42.2 & 51.7 \\
Sow & Finisher & 80.5 & 36.6 & 28.1 & 17.9 \\
Sow & Boar stud & 0.1 & 2.2 & 0.6 & 1.1 \\
Nursery & Sow & 7.3 & 5.8 & 4.9 & 4.6 \\
Nursery & Finisher & 27.5 & 33.7 & 10.9 & 16.6 \\
Nursery & Boar stud & 1.9 & 0.1 & 0.7 & 1.3 \\
Finisher & Sow & 18.9 & 10.9 & 14.1 & 5.3 \\
Finisher & Nursery & 12 & 16.7 & 13.2 & 6.4 \\
Finisher & Boar stud & 0.2 & 1.4 & 0.1 & 0.6 \\
Boar stud & Sow & 0.5 & 1.1 & 0.3 & 0.1 \\
Boar stud & Nursery & 3.9 & 2.5 & 2.1 & 2.4 \\
Boar stud & Finisher & 0.4 & 2 & 0.8 & 1.5 \\
\hline
\end{tabular}

853 Table 3. Number of transmission events that have been originated in farms that belong to the same pig

854 system (local) compared to the events coming from farms integrated into another pig system (external).

\begin{tabular}{ccccccc}
\hline \multirow{2}{*}{ System } & \multirow{2}{*}{ Year } & Events & \% & Events & \% & Total \\
& 2014 & 349 & $25.9 \%$ & 996 & $74.1 \%$ & 1345 \\
& 2015 & 294 & $25.9 \%$ & 840 & $74.1 \%$ & 1134 \\
\multirow{3}{*}{$\mathrm{A}$} & 2016 & 292 & $25.8 \%$ & 839 & $74.2 \%$ & 1131 \\
& 2017 & 330 & $28.3 \%$ & 836 & $71.7 \%$ & 1166 \\
& 2018 & 109 & $18.0 \%$ & 498 & $82.0 \%$ & 607 \\
\hline \multirow{3}{*}{$\mathrm{B}$} & 2014 & 299 & $22.1 \%$ & 1055 & $77.9 \%$ & 1354 \\
& 2015 & 310 & $27.5 \%$ & 819 & $72.5 \%$ & 1129 \\
& 2016 & 262 & $23.1 \%$ & 871 & $76.9 \%$ & 1133 \\
& 2017 & 205 & $17.6 \%$ & 963 & $82.4 \%$ & 1168 \\
& 2018 & 117 & $18.5 \%$ & 517 & $81.5 \%$ & 634 \\
\hline \multirow{3}{*}{$\mathrm{C}$} & 2014 & 228 & $16.9 \%$ & 1125 & $83.1 \%$ & 1353 \\
& 2015 & 60 & $5.3 \%$ & 1075 & $94.7 \%$ & 1135 \\
& 2016 & 126 & $11.2 \%$ & 1002 & $88.8 \%$ & 1128 \\
& 2017 & 80 & $6.8 \%$ & 1088 & $93.2 \%$ & 1168 \\
& 2018 & 54 & $8.9 \%$ & 551 & $91.1 \%$ & 605 \\
\hline
\end{tabular}

855 


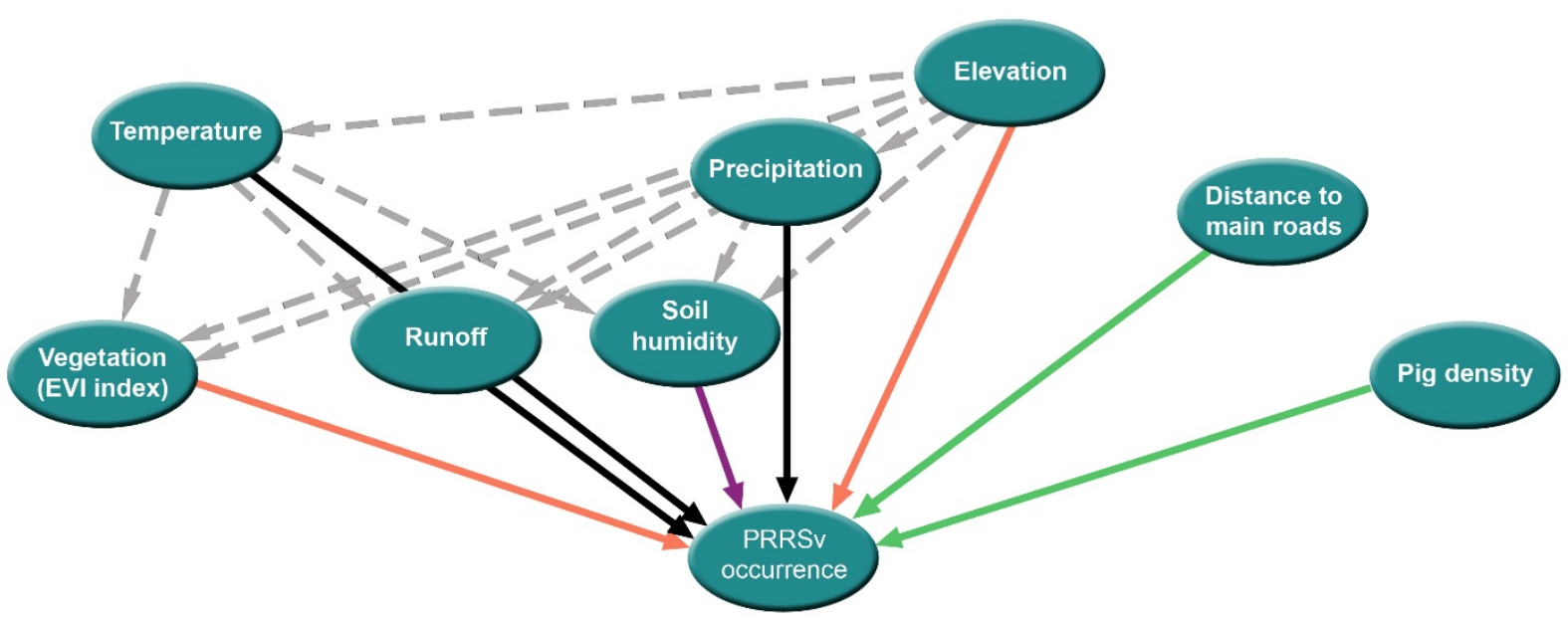

Figure 1. Diagram of factors promoting or restricting PRRSV spread. Arrows represent the relationship between the predictor and PRRSV occurrence based on Bayes factor analysis, where green and orange lines represent positive and negative drivers respectively. Black represent negligible support, and dashed lines show relatedness between environmental predictors affecting PRRSV through other variables. 


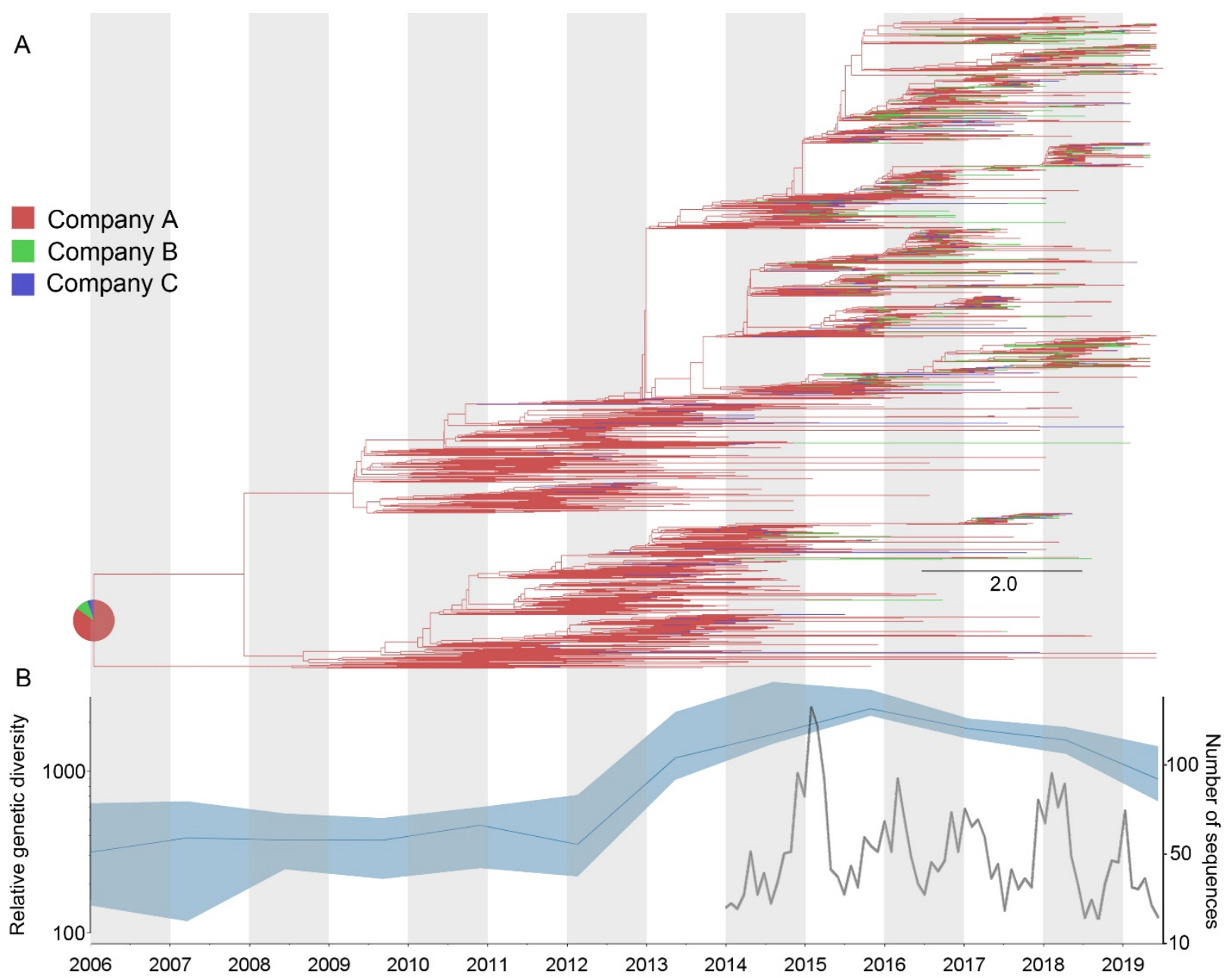

Figure 2. Dissemination history of PRRSV, inferred by discrete phylogeographic analysis. A) Maximum ancestral states (inferred from the Bayesian discrete trait analysis) are shown in pie charts on each node, representing the most likely type of farms. B) Spatiotemporal patterns in the relative genetic diversity

871 represented through the Bayesian SkyGrid plot and the number of sequences per month. The left y-axis

872 summarizes the effective population size over time, the mean estimate is represented by the dark blue

873 line, while the shaded light blue regions correspond to the $95 \%$ highest posterior density (HPD), and in

874 the right y-axis shows the number of sampled sequences over time. 


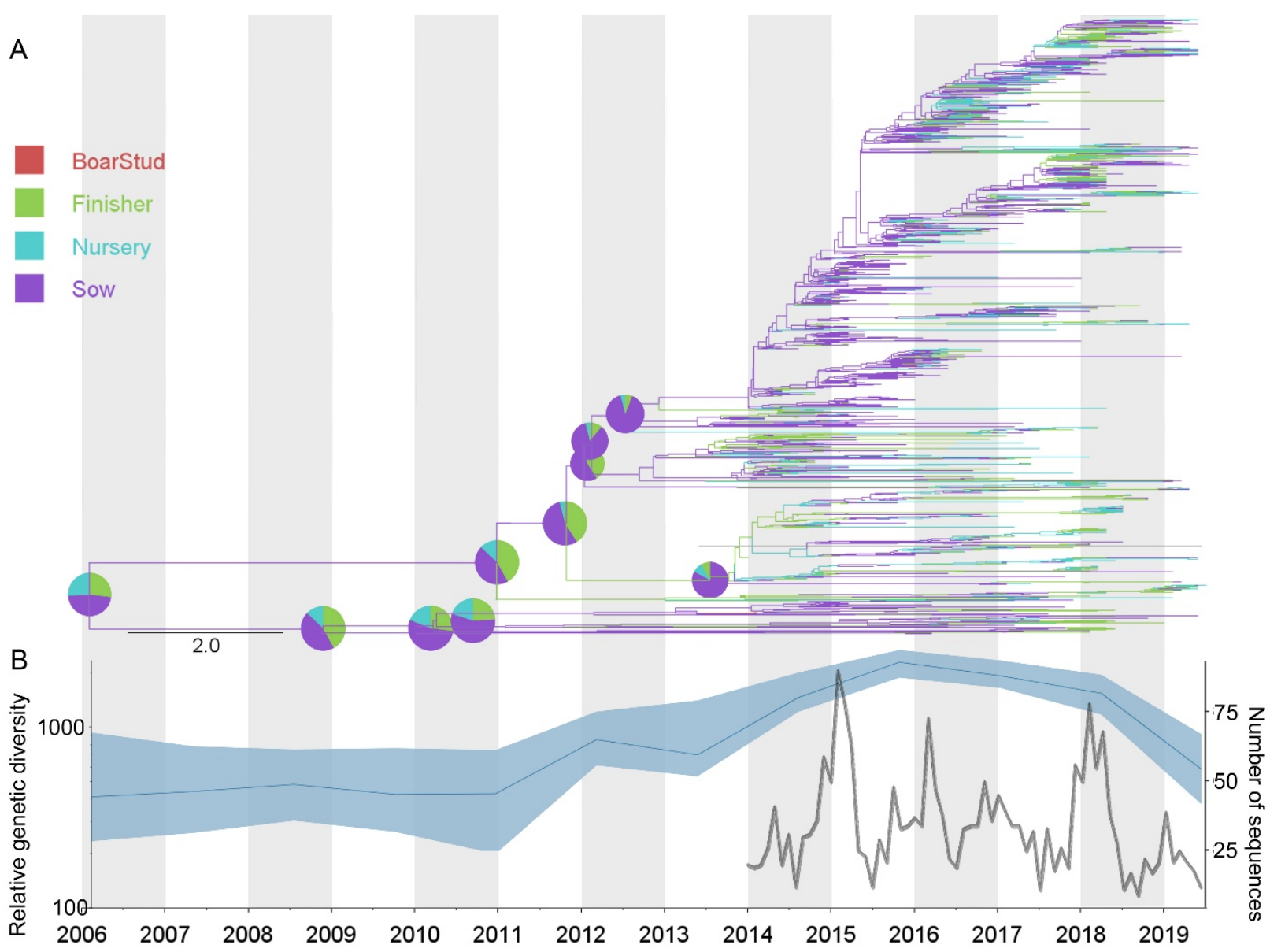

877 Figure 3. PRRSV dissemination within system A. Panel A) Maximum clade credibility phylogeny

878 colored according to the different farm types (sow, nursery, finisher, and boar stud). Probabilities of

879 ancestral states shown in pie charts in nodes, representing the most likely type of farm origin. Panel B)

880 Spatiotemporal patterns in the relative genetic variation through Bayesian SkyGrid plot and number of

881 sequences per month. The left y-axis summarizes the effective population size over time. Mean estimate

882 is represented by the dark blue line. Shaded light blue regions correspond to the $95 \%$ highest posterior

883 density (HPD), and in the right y-axis shows the number of sampled sequences over time. 


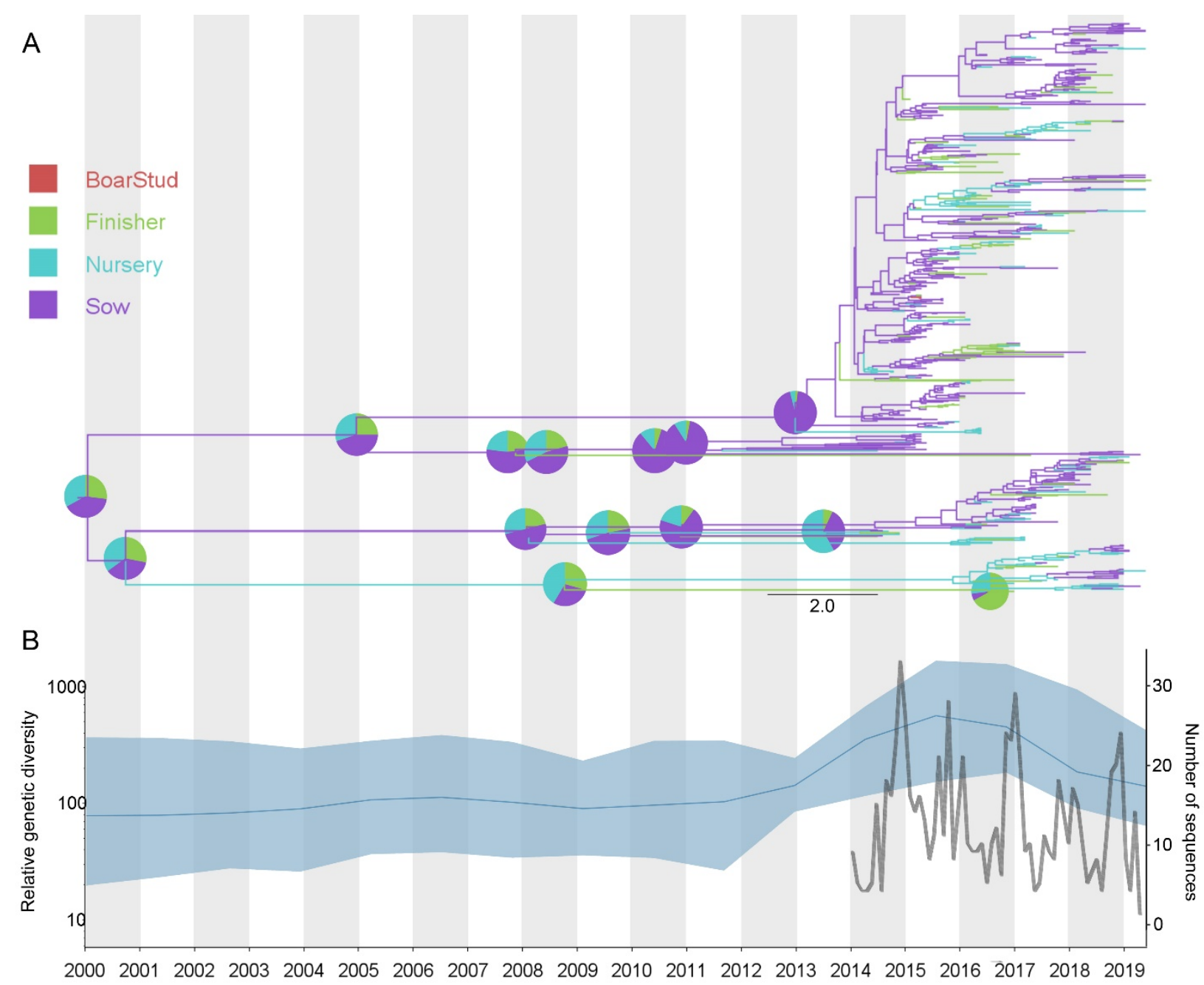

20002001200220032004200520062007200820092010201120122013201420152016201720182019

886 Figure 4. PRRSV dissemination within system B. Panel A) Maximum clade credibility phylogeny

887 colored according to the different farm types (sow, nursery, finisher, and boar stud). Probabilities of

888 ancestral states shown in pie charts in nodes, representing the most likely type of farm origin. Panel B)

889 Spatiotemporal patterns in the relative genetic variation through Bayesian SkyGrid plot and number of

890 sequences per month. The left y-axis summarizes the effective population size over time. Mean estimate

891 is represented by the dark blue line. Shaded light blue regions correspond to the $95 \%$ highest posterior

892 density (HPD), and in the right y-axis shows the number of sampled sequences over time. 
A

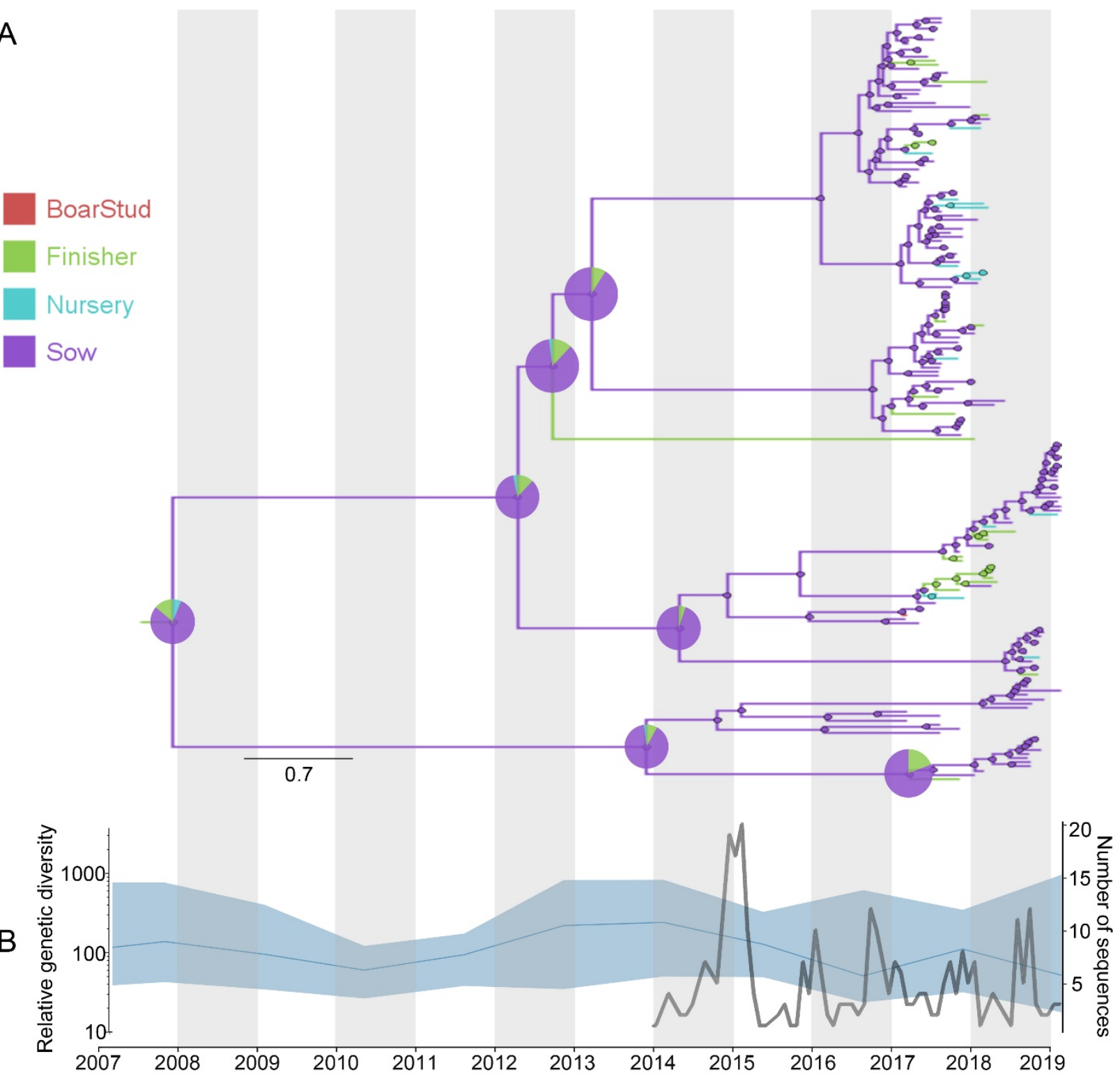

Figure 5. PRRSV dissemination within system C. Panel A) Maximum clade credibility phylogeny

colored according to the different farm types (sow, nursery, finisher, and boar stud). Probabilities of ancestral states shown in pie charts in nodes, representing the most likely type of farm origin. Panel B) Spatiotemporal patterns in the relative genetic variation through Bayesian SkyGrid plot and number of sequences per month. The left y-axis summarizes the effective population size over time. Mean estimate is represented by the dark blue line. Shaded light blue regions correspond to the $95 \%$ highest posterior

901 density (HPD), and in the right y-axis shows the number of sampled sequences over time. 


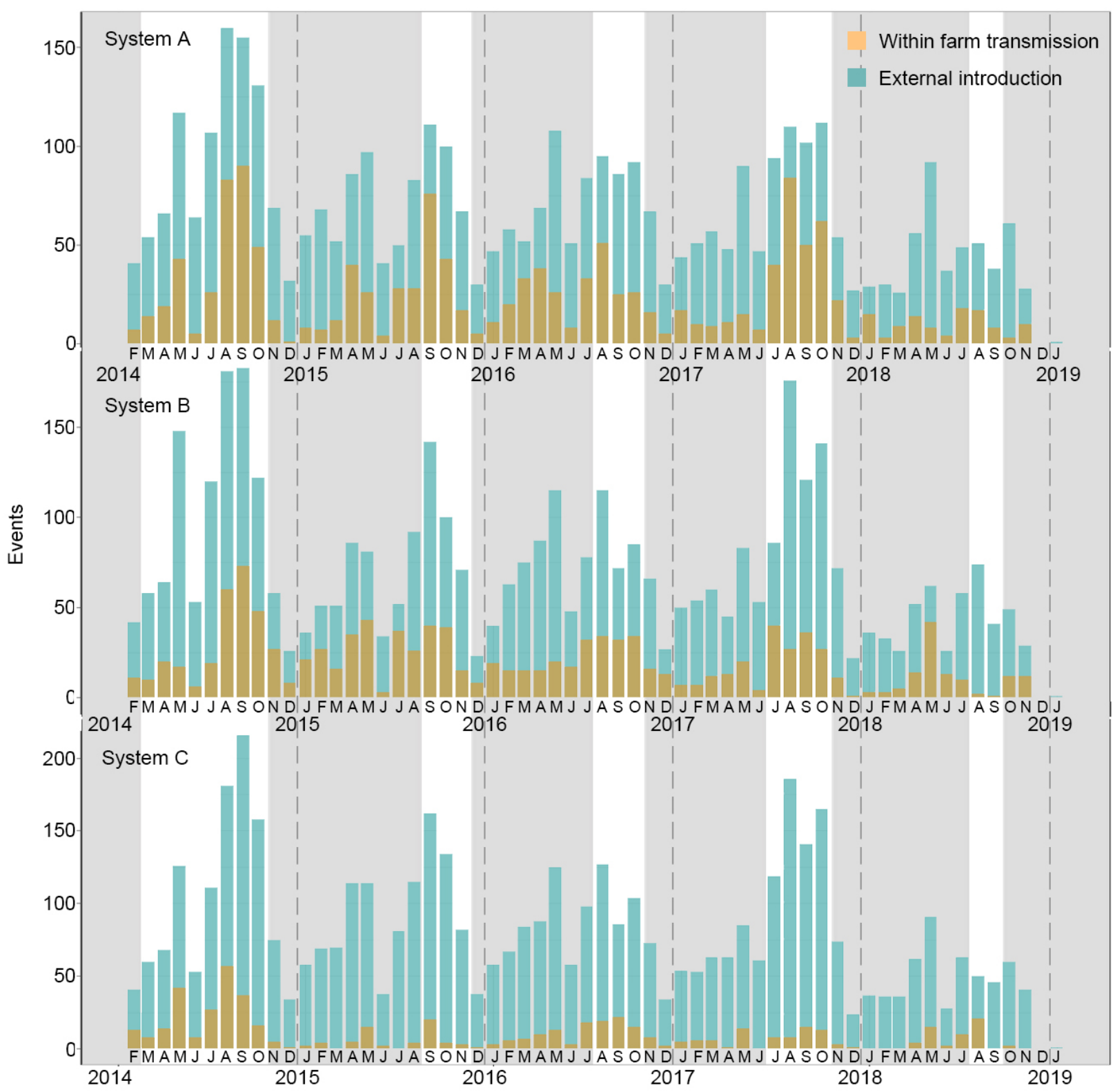

904 Figure 6. PRRSV migration events among pig systems are inferred through a structured coalescent

905 model. Number of dissemination events originated in farms from the same pig system compared to events

906 from farms integrated into another pig system. Grey background represents the months where the

907 exponentially weighted moving average epidemic monitor surpassed the established PRRSv epidemic

908 threshold based on the February 2020 MSHMP report (MSHMP, 2020), while dashed lines represent the

909 beginning of each year. 